\title{
The Cellular Location of the Streptococcal Group D Antigen
}

\author{
By G. D. SHOCKMAN AND H. D. SLADE \\ Departments of Microbiology, Temple University Medical School, \\ Philadelphia, U.S.A., and Northwestern University Medical School, \\ Chicago, U.S.A.
}

(Received 29 April 1964)

\section{SUMMARY}

The cellular location of the group $D$ antigen was examined by fractionation of protoplasts prepared by digestion of cell walls with lysozyme in an osmotically protective medium. The major portion of the group $D$ antigen appears to be closely associated with the protoplast membrane of Streptococcus faecalis (ATCC 9790). Some antigen was found in the supernatant fluid (lysozyme hydrolysate) after removal of the protoplasts. This was attributed to the known fragility of protoplasts and membranes.

\section{INTRODUCTION}

The development of good methods for the isolation of bacterial cell walls in relatively pure form has been followed by an accumulation of experimental evidence indicating that many of the antigens of Gram-positive bacteria are located in the cell-wall structure. The Lancefield streptococcal grouping antigens seem, in most instances, to be located in the wall. This has been demonstrated first by Salton (1953) for group A organisms, by Ottens \& Winkler (1962) for those belonging to group F and by Slade \& Slamp (1962 $a$ ) for organisms of groups B, C, E, F, G, H, K, L, M, $\mathrm{N}, \mathrm{O}$ and $\mathrm{Q}$. The group $\mathrm{D}$ streptococci therefore seem to be the one notable exception, in that the group specific antigen is not in the cell wall (Jones \& Shattock, 1960).

The grouping antigens of the streptococci have thus far been found to fall into two types of chemical substances. The group specific antigens of groups A and C appear to be rhamnose-containing polysaccharides (Krause \& McCarty, 1961, 1962). In groups $\mathbf{O}$ and $\mathrm{K}$ the same amino sugars are present, plus glucose and galactose; however, rhamnose may or may not be present (Slade \& Slamp, 1962 b). In contrast those from groups $\mathbf{D}$ and $\mathbf{N}$ appear to be glycerol-teichoic acids (Wicken, Elliott \& Baddiley, 1963; Elliott, 1962, 1963).

Recently we have reported a series of experiments which indicate that the group D antigen of an organism designated as Streptococcus faecalis ATCC 9790 by the American Type Culture Collection is closely associated with the protoplast membrane (Slade \& Shockman, $1963 a, b)$. The results of these experiments can be briefly summarized as follows: (1) complete disruption of either exponential phase or stationary phase cells by means of shaking with ballotini resulted in essentially all of the group $\mathbf{D}$ antigen remaining in the supernatant fluid after sedimentation at $10,000 \mathrm{~g}$. Centrifugation of the $10,000 \mathrm{~g}$ supernatant fluid at $105,000 \mathrm{~g}$ for $2 \mathrm{hr}$ (which will sediment fragmented membranes and, in the absence of added $\mathrm{Mg}^{2+}$, some of the ribosomes)

Vol. 37, No. 2 was issued 12 January 1965 
resulted in undetectable or barely detectable amounts of antigen in the supernatant fluid and large amounts of antigen in the pellet. (2) Isolated, well-washed (10 washings in sodium chloride or phosphate buffer) protoplast membranes from stationary phase, valine or threonine depleted cultures were obtained by lysozyme treatment of cells (Shockman et al. 1963). These membrane preparations contained relatively large amounts of the antigen. However, similar preparations from exponential phase cultures, washed 12 or 14 times, did not give a positive test for the antigen. The absence of antigen in the latter instances was attributed to the more extensive washing procedure which has been shown to remove antigen from the membranes (see below). However, a contributory factor might be the consistently lower titre of antigen observed with exponential phase cells and fractions from exponential phase cells as compared with those from any of the preparations from stationary phase cells. (3) Protoplasts of $S$. faecalis 9790 were prepared by digestion of the cell walls with lysozyme (lysozyme to cell ratio $1: 250$ to $1: 200$ in $0.5 \mathrm{M}$-sucrose $+\mathbf{0 . 0 5} \mathrm{M}$-phosphate buffer, $\mathrm{pH} \mathrm{7.4)}$. The development of osmotic fragility was followed by diluting samples 1 to 60 with water or the sucrose + phosphate solution. After maximum osmotic fragility had been reached, and the 3-12\% of the initial turbidity of the suspension that remained after dilution with water did not decrease any further with time, the entire protoplast suspension was lysed by dilution in $0.05 \mathrm{M}$-phosphate buffer. The membranes were sedimented and washed 8 times with sodium chloride or phosphate and for the 9th time with water on the centrifuge. All of these fractions (washed membranes, washes, and supernatant fluid) were dialysed and their content of group $\mathbf{D}$ antigen was determined by the capillary precipitin method. The washed membranes as well as all of the 9 washings (particularly the last water wash) gave strong precipitin reactions for the group $D$ antigen. Additional similar experiments with both exponential phase and stationary phase cells, in which the membrane fraction was washed only once with water, gave similar results. It was clear from these experiments that a considerable portion of the group D antigen was closely associated with the membrane but could be removed, at least partially, by washing with sodium chloride, phosphate buffer and especially with water.

However, at that time we were unable to get a supernatant fluid after digestion of whole cells by lysozyme (EC 3.2.1.17) in an osmotically-protected medium that we could be certain was reasonably free from whole cells as well as membrane and cytoplasmic contents. Therefore we were unable to examine the possibility of a cellular location of the antigen, external to the membrane but not part of the cell wall. On the other hand, Hijmans (1962) clearly showed that L-forms of three strains of group D streptococci did not contain the group antigen. In addition, since the completion of our previous experiments, two groups of workers have briefly reported evidence pointing to a location of the antigen in the region between the protoplast membrane and the cell wall. Shattock \& Smith (1963) examined several strains of Streptococcus faecalis by a variety of procedures such as (1) digestion of cell walls with lysozyme, (2) digestion of walls with a phage-associated lysin in osmotically protected media, and (3) the synthesis of the antigen by $\mathbf{L}$-forms. A gel diffusion precipitin method for detection of the antigen was used. They concluded that $50-85 \%$ of the group antigen was external to the protoplast membrane and that the Hijmans L-form can, upon proper treatment, continue to produce the group D 
antigen, $97 \%$ of which was found free in the culture medium. Also, Hay, Wicken \& Baddiley (1963), using paper chromatography and chemical methods to identify the hydrolysis products of teichoic acids after digestion of cell walls of a group D streptococcus (NCTC 8191) with lysozyme suspended in an osmotically protective medium, showed ' ... that at least $85 \%$ of the glycerol teichoic acid was present in the wall hydrolysate'. These investigators interpreted these findings as indicating that the so-called intracellular glycerol-teichoic acid is 'probably located in a region between the wall and the protoplast membrane, perhaps in weak association with the latter'.

Thus an area of doubt remained. Therefore attempts were made to increase the osmotic stability of our protoplasts while still insuring essentially complete removal of the cell wall so that the supernatant fluid after digestion of the walls with lysozyme could be obtained relatively free from contamination with other cellular fractions. We were able to do this by making carefully controlled lysozyme digestions in the presence of $\mathrm{Mg}^{2+}$ which has been shown by Weibull (1956) to increase the stability of protoplasts. Preliminary experiments indicated that $10^{-2} \mathrm{M}$-magnesium acetate was the most suitable concentration. These protoplasts are far more stable than are those made in the absence of $\mathbf{M g}^{2+}$ and in fact lyse more slowly after dilution with water or $0.05 \mathrm{M}$-phosphate buffer.

\section{METHODS}

Organisms. The strain designated as Streptococcus faecalis ATCC 9790 was used throughout these studies. This is a group D streptococcus which is atypical of the $S$. faecalis species designation (Slade \& Shockman, $1963 b$; Chesbro, 1961) and perhaps could more properly be designated as $S$. faecium. This strain has the advantage that the cell wall can be digested by lysozyme (Abrams, 1958). With appropriate conditions wall-free protoplasts can be obtained (Shockman et al. 1961, 1963).

Conditions for growth and harvest. Cultures were grown in a completely defined medium under conditions of valine limitation and harvested as previously described (Slade \& Shockman, 1963b; Shockman et al. 1963).

Serological procedures. All serological procedures were as previously described (Slade \& Shockman, $1963 b$ ). The capillary procedure of Swift, Wilson \& Lancefield (1943) was used, starting with extracts containing $1 \mathrm{mg}$. dry weight $/ \mathrm{ml}$. The group D antigen was extracted from dialysed samples with $0.5 \mathrm{~N}-\mathrm{HCl}$ at $100^{\circ}$ for $10 \mathrm{~min}$. and neutralized to phenol red with $\mathrm{NaOH}$. The specificity of the precipitin reaction for the group D antigen has previously been determined (Slade \& Shockman, 1963b). A single batch (lot no. 2) of antiserum obtained from the U.S. Public Health Service was used throughout.

Preparation of protoplasts and fractionation procedures. All operations, where possible, were carried out in the cold and followed by phase contrast microscopy. Cocci were grown in 1.21 . of the defined medium containing $13 \mu \mathrm{g}$. $\mathrm{L}$-valine $/ \mathrm{ml}$. for $17-20 \mathrm{hr}$ at $38^{\circ}$ harvested and washed by centrifugation. Approximately $600 \mathrm{mg}$. (dry weight) of cocci were suspended in $20 \mathrm{ml}$. of $0.5 \mathrm{M}$-sucrose, $0.01 \mathrm{M}$ magnesium acetate, $0.05 \mathrm{M}$-sodium phosphate buffer ( $\mathrm{pH} \mathrm{6.6)}$ ) and $2 \mathrm{mg}$. lysozyme (lysozyme to cell ratio $1: 333$ ) and incubated at $38^{\circ}$ (Shockman et al. 1963). Osmotic fragility was followed by dilution of $0.1 \mathrm{ml}$. samples to $6 \mathrm{ml}$. with water. At this low ratio of lysozyme to cocci, maximum osmotic fragility was reached in about $11 \mathrm{hr}$ 
in Expt. 1, Table 1. (When diluted with water, $7 \%$ of the turbidity at time 0 remained.) Incubation was allowed to proceed for an additional hour to be certain that maximum osmotic fragility was obtained. Phase-contrast microscopy showed single spherical bodies which could be observed under the microscope to swell and burst upon dilution of the $0.5 \mathrm{M}$-sucrose. After dilution of the sucrose, only empty 'ghosts' could be seen. Streptococcal forms were only very rarely observed in these preparations. Whole protoplasts or streptococci would be far more easily seen in the microscope than are the membrane 'ghosts'. A sample was removed (total) and the remainder was centrifuged at $10,000 \mathrm{~g}$ for $20 \mathrm{~min}$. The supernatant fluid (lysozyme hydrolysate) was decanted. It did not prove to be possible to resuspend these protoplasts without significant disruption. The protoplasts were then disrupted by dilution to $250 \mathrm{ml}$. with water containing $1 \mathrm{mg}$. DNAase. A sample was removed (protoplast lysate) and, after adequate time to insure complete disruption, the remainder was centrifuged at $\mathbf{2 5 , 0 0 0} \mathrm{g}$ for $2 \mathrm{hr}$. The pelleted crude membranes were washed once with water and recentrifuged as above. A portion of the lysozyme hydrolysate was centrifuged at $120,000 \mathrm{~g}$ for $2 \mathrm{hr}$.

A subsequent batch of protoplasts (Exp. 2, Table 1) was prepared using the extremely high lysozyme to cell ratio of $1: 2 \cdot 5$ and a 3 -hr incubation period. Maximum osmotic fragility was reached in about $2 \mathrm{hr}$, at which time, when diluted with water about $30 \%$ of the turbidity at time 0 still remained. Microscopic examination showed the presence of very small spherical bodies. These protoplasts were extremely stable and, in fact, were difficult to lyse by 1 to 60 dilution with water. However, they did lyse completely in about $40 \mathrm{~min}$. after centrifugation and resuspension in $250 \mathrm{ml}$. of water (about $8 \%$ of the initial turbidity remained).

The release of nucleic acids and derivatives was followed by measuring their ultraviolet absorption and comparing the $260 \mathrm{~m} \mu$ absorption peaks of the various fractions.

\section{RESULTS}

Table 1 shows the results of two typical experiments. Clearly, the once-washed protoplast membrane fraction (line 4) reacts with the group D antiserum to a high titre. Some antigen is present in the wash (line 5). Our previous experiments (Slade \& Shockman, 1963b) showed that subsequent washings in water, buffer, or in $\mathrm{NaCl}$ continued to remove antigen from the membrane. However, with similar cells considerable amounts of antigen remained with the membranes after 10 to 12 washings. It is equally clear that antigen was present in the lysozyme hydrolysate (line 6). A portion of this activity could be sedimented at $120,000 \mathrm{~g}$. From the estimated percentage of total cell substance that each fraction represents (last column of Table 1 ) it is clear that the membrane fraction (27\%) represents far more cell substance than does the $120,000 \mathrm{~g}$ pellet of the lysozyme hydrolysate $(5 \%)$. The percentage of total cell substance represented by this fraction was directly determined from this experiment on dialysed samples. Therefore, since at least a portion of the supernatant fluid of the lysozyme hydrolysate should be dialysable (Salton 1956, 1960), the estimate of $5 \%$ for the pellet should be viewed as a maximum. These titres of antigen could easily have been derived from membrane contamination resulting from a small amount of protoplast breakage during the preparative procedure or even to the presence of a few whole cocci which, if present, would concentrate in this fraction. 
Streptococcal group $D$ antigen

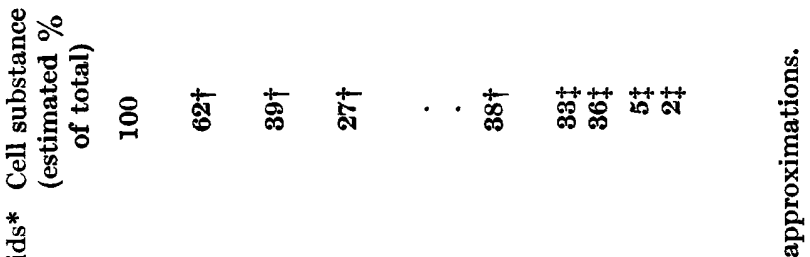

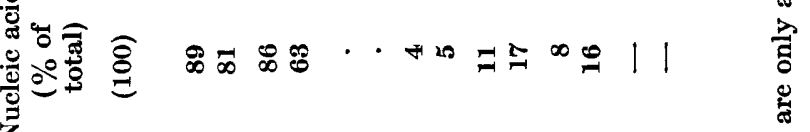

:

$\int \ldots, \ldots, \ldots \ldots$

है

$\theta$

2

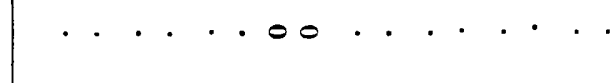

$.000 .00+.0 .0 .0 .0$

要

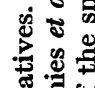

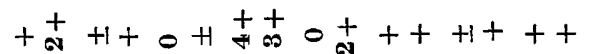

远

ن.

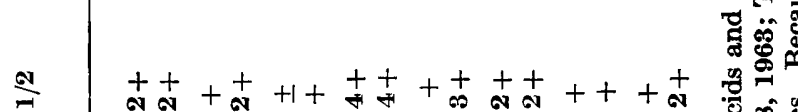

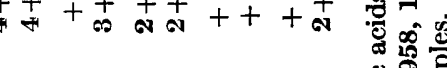

월

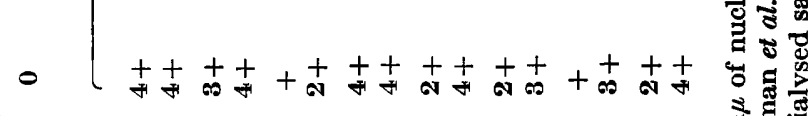

氠

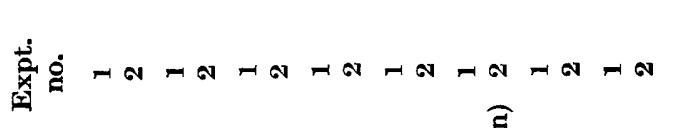

要

$\overrightarrow{\mathrm{w}}$ के

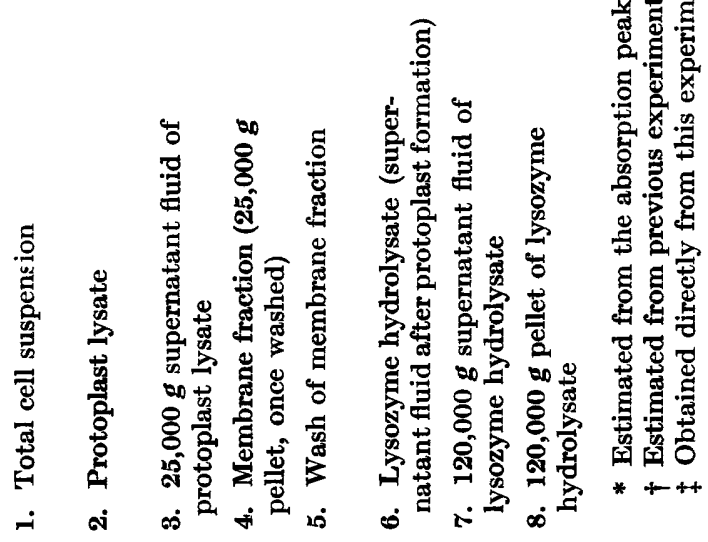


The precipitin reactions are based on dilutions of antigen $1 \mathrm{mg} / \mathrm{ml}$. We can then conclude that the antigen in the membrane fraction is largely responsible for the antigen titres extracted from the total cell suspension.

Since these results are in disagreement with those in the literature (Hay et al. 1963; Shattock \& Smith, 1963) reasons for this disagreement were sought. Since lysozyme is known to be bound by membranes, particularly when present at high concentrations (Weibull et al. 1959; Shockman et al. 1963) and since at least one of the reports with which we are in disagreement (Hay et al. 1963) used a high ratio of lysozyme to cells, this point was investigated further. Two mg. of the membrane fraction from Expt. 1, Table 1, was treated with $2 \mathrm{mg}$. lysozyme in $0.05 \mathrm{M}$ phosphate buffer ( $\mathrm{pH} 7 \cdot 4)$ at $38^{\circ}$ for $2.5 \mathrm{hr}$. No precipitin reaction was obtained with the supernatant fluid either before or after dialysis.

Since the result of this experiment was negative, an additional isolation experiment, essentially identical to the one previously described, but using a higher lysozyme to cell ratio $(1: 2 \cdot 5)$ and a shorter time period for wall digestion, was performed. The results are shown in Table 1 (Expt. 2) and are in essence the same as. those for the first experiment. For unknown reasons a somewhat less clean fractionation was obtained in Expt. 2 as shown by the higher percentage of total cellular nucleic acid which appeared in the lysozyme hydrolysate.

\section{DISCUSSION}

It is clear from these results that the major portion of the glycerol-teichoic acid group $\mathrm{D}$ antigen in our strain of enterococcus does not seem to be located between the cell wall and the protoplast membrane. The results of the experiments reported here confirm and closely correlate with our previous results (Slade \& Shockman, $1963 \mathrm{~b}$ ) which were interpreted to indicate a close association of the antigen with the protoplast membrane. The experiments reported by both Shattock \& Smith (1963) and Hay et al. (1963) with different strains of Group D streptococci are in disagreement with our findings. Hay et al. (1963) used determinations of glycerolteichoic acid by chemical methods on paper chromatograms. No determinations of antigen by the use of antibody were made. In at least some group $\mathrm{D}$ organisms there may be other glycerol-teichoic acids present in addition to the group antigen. The fragility of protoplasts and protoplast membranes are definitely complicating factors for an unequivocal interpretation of the origin of the antigenic activity found in the lysozyme hydrolysate of the cell wall. We believe that adequate, although not complete, control of these factors was obtained in the experiments reported. Release of $260 \mathrm{~m} \mu$ absorbing substances to the supernatant fluid is only a limited and relatively crude measure of damage of the permeability barrier. Control of the fragility of the membranes themselves is more difficult and was evaded in. these experiments by simply washing the membrane fraction only once. Our previous series of experiments (Slade \& Shockman, 1963 $a, b$ ) provided evidence indicating that a large portion of the antigen remains with the membranes of these cells after extensive washings and that release of the antigen from the membrane by washing does not correlate with release of $260 \mathrm{~m} \mu$ absorbing substances. However, during the washings the membranes do tend to fragment and it is difficult to differentiate between lack of sedimentation of membrane fragments and selective 'elution' of the antigen from the membranes. 
Valine-deprived cocci were selected for specific reasons. Cocci grown in this manner seem to contain higher titres of antigen (unpublished observations) as well as more membrane substance (Shockman et al. 1963; Toennies et al. 1963) than exponentially growing cultures. Other investigators (Medrek \& Barnes, 1962) have shown that nutritional and physiological conditions can influence the amount of group antigen extracted from several group D streptococci. Growth conditions resulting in decreased antigen yield might easily influence the observed cellular distribution of the antigens. Also, valine deprived cultures are not prone to autolysis (Shockman et al. 1961). Partial autolysis of cocci during harvesting or incubation in buffer might easily further complicate the picture.

Since the completion of these experiments an article by Smith \& Shattock (1964) has appeared which presents data in support of their previous conclusion (Shattock $\&$ Smith, 1963) that the group $D$ antigen is located between the protoplast membrane and the cell wall. On the basis of the greatest dilution of antigen extracts which showed reaction with antiserum in a slide gel-diffusion test, these investigators found that $80-90 \%$ of the group $\mathrm{D}$ antigen went into solution during protoplast formation as a result of treatment with a phage-associated lysin. Since neither the capillary precipitin method that we used, or the gel-diffusion test used by Smith \& Shattock (1964) was quantitated (for example, on the basis of precipitated protein or nitrogen), we have taken the more conservative view and have expressed our results in a relative manner.

Smith \& Shattock (1964) ' . . concluded that the organism received as ATcc 9790 was an unsuitable strain for investigating the location of the group $\mathbf{D}$ antigen'. They examined two cultures of this strain, one received from Dr A. Abrams and one from the National Collection of Industrial Bacteria, and found that they reacted only weakly with group D antiserum. These investigators therefore used other group D strains for their investigations. We were able to obtain strong precipitin reactions with the ATCC 9790 strain, and with the various cellular fractions from this strain, using group specific antisera prepared against four strains of group D streptococci (S. liquefaciens (strain 8175), S. zymogenes (strain 8176), S. faecalis (strain 8213) and $S$. durans (strain 8307)), which represent members of each of the four major groups of streptococci of group D. The results obtained using these four antisera correlated completely with those obtained using USPHS lot no. 2 antiserum (Slade \& Shockman, $1963 b$ ). Perhaps the failure of Smith \& Shattock to obtain satisfactory titres with the ATCC 9790 strain can be explained by their use of exponentially growing cultures as starting material. Exponential phase cultures of ATcc 9790 contain less antigen than do stationary phase cultures (Slade \& Shockman, 1963 $b$, unpublished observations), are prone to autolysis (Shockman et al. 1961) and possess membranes of extreme fragility (Slade \& Shockman, 1963; Shockman et al. 1963; above discussion).

Smith \& Shattock were also unable to obtain osmotically-fragile bodies that looked like spherical protoplasts after treatment of other group D streptococci with lysozyme. This is in contrast with our previous results and to those of Abrams (1959) with ATCC 9790 and to those of Chesbro (1961) with a variety of group D strains. Chesbro observed a spectrum of lysozyme sensitivity from resistant to completesensitivity with a variety of group D strains. As noted above (Expt. 2) a relatively high ratio of lysozyme to cocci $(1: \mathbf{2 \cdot 5})$ can result in protoplasts that are difficult to lyse. 
Smith \& Shattock did not present data concerning the osmotic stability of their protoplast or spheroplast preparations. It is surprising that these investigators made no attempt to increase stability of membranes of spherical bodies such as by the addition of $\mathrm{Mg}^{2+}$. The known and previously mentioned fragility of membranes plus the ease of removal of the antigen from membranes by washing procedures could easily account for the release of group $\mathbf{D}$ antigen to the supernatant fluid during treatment with lysozyme or phage-associated lysin. While it is true that Smith \& Shattock used a different group D streptococcus from the one that we used, their experiments did not eliminate the possibility of removal of antigen from the membrane during their experimental procedures.

A hypothetical location of the glycerol-teichoic acid group $\mathbf{D}$ antigen on or in the external surface of the protoplast membrane would be consistent not only with our results but also with those of Smith \& Shattock, and those of Hay, Wicken \& Baddiley. Further resolution of this problem might be materially aided when some information concerning the biological role of glycerol-teichoic acids becomes. available.

This work was supported by the National Institute of Allergy and Infectious Diseases (AI 5044-01), and the National Heart Institute (H-3709) of the National Institutes of Health, United States Public Health Service, the Chicago Heart Association, the Office of Naval Research, Department of the Navy (Nonr-1768), and National Science Foundation (GB-856). H. D. S. is a Research Career Awardee of the National Institutes of Health. The authors wish to thank Miss M. J. Conover and $\mathrm{Mr}$ W. Slamp for excellent technical assistance.

\section{REFERENCES}

Abrams, A. (1958). O-acetyl groups in the cell wall of Streptococcus faecalis. J. biol. Chem. 230, 949.

Aвrams, A. (1959). Reversible metabolic swelling of bacterial protoplasts. J. biol. Chem. 234, 383.

Cressro, W. R. (1961). Lysozyme and the production of osmotic fragility in enterococci. Canad. J. Microbiol. 7, 952.

Elliotr, S. D. (1962). Teichoic acid and the group antigen of group D streptococei. Nature, Lond. 193, 1105.

Elliotr, S. D. (1963). Teichoic acid and the group antigen of lactic streptococci (Group N). Nature, Lond. 200, 1184.

Hay, J. B., Wicken, A. J. \& Baddiuey, J. (1963). The location of intracellular teichoic acids. Biochim. biophys. Acta, 71, 188.

Hismans, W. (1962). Absence of the group-specific and the cell-wall polysaccharide antigen in L-phase variants of group D streptococci. J. gen. Micriobiol. 28, 177.

Jones, D. \& Shatrock, P. M. F. (1960). The location of the group antigen of Group D streptococcus. J. gen. Microbiol. 23, 335.

Krause, R. M. \& MCCARTY, M. (1961). Studies on the chemical structure of the streptococcal cell wall. I. The identification of a mucopeptide in the cell walls of group $A$ and A-variant streptococci. J. exp. Med. 114, 127.

Krause, R. M. \& McCarty, M. (1962). Studies on the chemical structure of the streptococcal cell wall. II. The composition of group $\mathrm{C}$ cell walls and chemical basis for serologic specificity of the carbohydrate moiety. J. exp. Med. 115, 49.

MEDREK, T. F. \& BARnes, E. M. (1962). The influence of the growth medium on the demonstration of a group D antigen in faecal streptococci. J. gen. Microbiol. 28, 701. 
OTtens, H. \& WiNKuER, K. C. (1962). Indifferent and haemolytic streptococci possessing group-antigen F. J. gen. Microbiol. 28, 181.

Salton, M. R. J. (1953). The action of lysozyme on cell walls of some lysozyme sensitive bacteria. Biochim. biophys. Acta, 22, 495.

Salton, M. R. J. (1960). Microbial Cell Walls. New York: John Wiley and Sons.

Shatrock, P. M. F. \& Smrth, D. G. (1963). The location of the group D antigen in a strain of Streptococcus faecalis var. liquefaciens. J. gen. Microbiol. 31, iv.

Shockman, G. D., Conover, M. J., Kolb, J. J., Phillips, P. P., Riley, L. S. \& Toennies, G. (1961). Lysis of Streptococcus faecalis. J. Bact. 81, 36.

Shockman, G. D., Kolb, J. J., Bakay, B., Conover, M. J. \& Toennies, G. (1963). Protoplast membrane of Streptococcus faecalis. J. Bact. 85, 168.

Slade, H. D. \& Shockman, G. D. $(1963 a)$. The protoplast membrane and the D antigen of Streptococcus faecalis. Bact. Proc. p. 46.

Slade, H. D. \& Shockman, G. D. (1963b). The protoplast membrane and the group D antigen of Streptococcus faecalis. Iorwa St. Coll. J. Sci. 38, 83.

Slade, H. D. \& Slamp, W. C. (1962a). Cell-wall composition and the grouping antigens of streptococci. J. Bact. 84, 345.

Slade, H. D. \& Slamp, W. C. (1962b). Chemical composition of streptococcal cell walls. Bact. Proc. p. 62.

Smith, D. G. \& Shatтock, P. M. F. (1964). The cellular location of antigens in streptococci of groups $\mathrm{D}, \mathrm{N}$ and $\mathrm{Q}$. J. gen. Microbiol. 34, 165 .

SwifT, H. F., Wilson, A. T. \& Lancefield, R. (1943). Typing group A hemolytic streptococci by $M$ precipitin reactions in capillary pipettes. J. exp. Med. 78, 127.

Toennies, G., Shockman, G. D. \& Kolb, J. J. (1963). Differential effects of amino acid deficiencies on bacterial cytochemistry. Biochemistry, N.Y. 2, 294.

Weibull, C. (1956). The nature of the 'ghosts' obtained by lysozyme lysis of Bacillus megaterium. Exp. Cell Res. 10, 214.

Weibull, C., Zacharias, B. \& Beckman, H. (1959). Affinity of lysozyme to structural elements of the bacterial cell as studied with enzymes labelled with radioactive iodine. Nature, Lond. 184, 1744.

Wicken, A. J., Elliott, S. D. \& Baddiley, J. (1963). The identity of streptococcal group D antigen with teichoic acid. J. gen. Microbiol. 31, 231. 